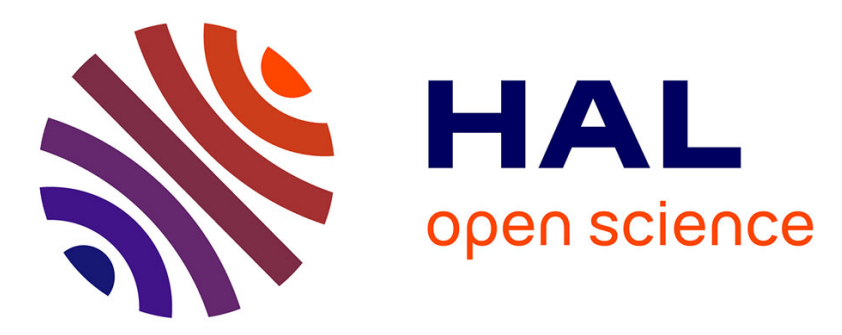

\title{
Le rôle des Parties Prenantes dans la gouvernance de la conception des plans communaux de développement au Maroc
}

\author{
Ekaterina Le Pennec, Marc Olivaux
}

\section{To cite this version:}

Ekaterina Le Pennec, Marc Olivaux. Le rôle des Parties Prenantes dans la gouvernance de la conception des plans communaux de développement au Maroc. Maghreb-Machrek, 2014, Gouvernance territoriale au Maroc, 219. hal-01826323

\section{HAL Id: hal-01826323 \\ https://hal.science/hal-01826323}

Submitted on 29 Jun 2018

HAL is a multi-disciplinary open access archive for the deposit and dissemination of scientific research documents, whether they are published or not. The documents may come from teaching and research institutions in France or abroad, or from public or private research centers.
L'archive ouverte pluridisciplinaire HAL, est destinée au dépôt et à la diffusion de documents scientifiques de niveau recherche, publiés ou non, émanant des établissements d'enseignement et de recherche français ou étrangers, des laboratoires publics ou privés. 


\title{
AIX-MARSEILLE UNIVERSITÉ \\ Centre d'études et recherches en gestion d'Aix-Marseille (CERGAM) \\ Institut de Management Public et Gouvernance Territoriale (IMPGT)
}

\section{«Le rôle des Parties Prenantes dans la gouvernance de la conception des plans communaux de développement au Maroc »}

\author{
Ekaterina Le Pennec \\ ATER de l'Institut de Management Public et Gouvernance Territoriale \\ (Université d'Aix-Marseille) \\ Doctorante de l'IAE de Nice \\ @: lepennecekaterina@gmail.com \\ Marc Olivaux \\ Allocataire de Recherche Chargé de Mission d'Enseignement \\ IMPGT - Université d'Aix-Marseille \\ @: marc.olivaux@univ-amu.fr ${ }^{1}$
}

Mots clefs : développement territorial, gouvernance, parties prenantes, théorie de l'acteurréseau, entretien d'experts, formation

\section{Résumé :}

L'objectif de cette recherche est d'analyser l'implication des parties prenantes dans la conception des plans communaux de développement au Maroc. L'originalité de ce projet réside dans la combinaison des modèles traditionnels d'analyse du positionnement des parties prenantes avec une analyse du processus de mobilisation à l'aide de la théorie de l'acteurréseau. Notre analyse, à destination des pouvoirs publics, a pour objet d'aider à l'amélioration de la gestion des processus de développement territorial. Au delà d'une simple analyse de l'implication des acteurs, ce travail met également en évidence d'autres éléments ayant émergé du terrain tels que les besoins de formation des élus locaux aux logiques de développement local.

\begin{abstract}
:
The aim of this research is the analysis of stakeholders' involvement in the conception of Communal Development Plans in Morocco. The originality of this project lies in combination of two traditional models, stakeholder theory and the actor-network theory. Our analysis and recommendations are addressed to Public Sector Authorities and Agencies for improvement efforts in management practices. Beyond a simple analysis of the actors' implication, this work also highlights elements having emerged from the field such as the needs for training of the local elected representatives to the logics of local development.
\end{abstract}

\footnotetext{
${ }^{1}$ Authors appear in alphabetic order
} 


\section{Introduction}

Ce travail a pour objectif d'analyser la conception des plans communaux de développement (PCD) au Maroc afin d'observer l'implication des parties prenantes dans ce processus et leurs rôles. En nous appuyant sur différentes typologies de positionnement des parties prenantes et sur la théorie de l'acteur-réseau, nous conceptualisons le processus d'implication de ces parties prenantes à partir d'entretiens d'experts réalisés avec des élus locaux et des spécialistes des questions de développement local au Maroc (universitaires, bureaux d'études, etc.).

Les parties prenantes peuvent être définies comme " tout groupe ou individu qui affecte ou est affecté par la réalisation des objectifs de l'entreprise » (Freeman, 1984²). Pour comprendre et analyser le processus d'implication des parties prenantes, nous avons centré notre analyse sur les questions suivantes :

Quelles sont les parties prenantes impliquées ? Comment et pourquoi ont-elles été choisies ? Et enfin, quel est le rôle de ces derniers dans la conception des plans communaux ?

Afin de mieux répondre à ces questions nous allons, dans un premier temps, présenter le concept de gouvernance territoriale ${ }^{3,4}$. Nous sommes particulièrement intéressés par la théorie dominante concernant l'organisation des processus de développement dans le monde à savoir le paradigme utilitariste de la « bonne gouvernance $»^{5}$. Ensuite nous allons développer le concept des parties prenantes (Mitchell, Agle et Wood, $1997^{6}$; Girard et Sobczak, 2010 ${ }^{7}$ ) mais aussi la théorie de l'acteur-réseau qui s'intéresse aux processus de mobilisation des acteurs (Callon, $1988^{8}$ ).

Dans un deuxième temps nous développons la méthodologie utilisée au travers d'une présentation de l'étude de cas. Notre problématique de recherche, étant relativement peu traitée dans la littérature, une étude qualitative exploratoire est préférable puisqu'elle

\footnotetext{
2 Freeman (R. E.), Strategic Management: A Stakeholder Approach, Pitman, Boston, 1984

3 Clarke (T.), Theories of corporate governance, The philosophical foundations of corporate governance, London \& New-York Routledge, 2004

${ }^{4}$ Guenoun (M.), Le management de la performance publique locale, Etude de l'utilisation des outils de gestion dans deux organisations intercommunales, Thèse de Doctorat, Université Paul Cézanne Aix-Marseille III, 2009

5 Bessire (D.), Chatelin \& Onnée, «Qu'est-ce qu'une bonne gouvernance ? », Comptabilité et environnement, 2007.

${ }^{6}$ Mitchell (R. K.), Agle (B. R.) \& Wood (D. J.), "Toward a Theory of Stakeholder Identification and Salience: Defining the Principle of Who and What Really Counts", $A M R$, vol. 22, n 4, 1997, pp. 853-886.

${ }^{7}$ Girard (C.) \& Sobczak (A.), "Pour une cartographie des parties prenantes fondée sur leur engagement : une application aux sociétaires d'une banque mutualiste française", Management et Avenir, N 33, 2010, pp.157-174.

${ }^{8}$ Callon, (M.) (dir.), La science et ses réseaux. Genèse et circulation des faits scientifiques, Paris : La Découverte, 1988
} 
permettra de saisir toute la richesse des données (Bédard et Gendron, 2004 ${ }^{9}$; Ahrens et Chapman, $2006^{10}$ ). La méthode de recueil des données retenue est l'entretien d'expert. Au nombre de huit, ces entretiens seront analysés textuellement. Pour conclure, nous proposerons une évaluation du cadre d'analyse appliqué ainsi qu'un exposé des limites de notre étude.

Nous avons choisi le Maroc comme terrain de recherche en raison de la dynamique de développement local qui y a été engagée depuis une décennie. En effet, depuis l'adoption de la Charte Communale en 2002 et, dans son prolongement, depuis l'Initiative Nationale pour le Développement Humain lancée par SM le Roi Mohammed VI en 2005, chaque commune marocaine est légalement contrainte de se doter d'un Plan Communal de Développement afin d'envisager son avenir en termes de développement local en co-construisant avec les acteurs de terrain et la société civile des plans de développement locaux adaptés tant à leurs attentes qu'aux spécificités du terrain. C'est en raison tant du caractère assez récent de ces processus que des nombreuses situations d'échec dans leur mise en oeuvre que nous avons choisi ce terrain. D'une part, le sujet est à la fois assez novateur pour ne jamais avoir été traité sous le prisme de la théorie de l'acteur-réseau, mais également assez ancien pour que les experts interrogés aient un vrai recul sur la situation. D'autre part, cette recherche constitue la première étape d'une démarche visant à identifier les causes de dysfonctionnements des processus afin d'aider à y apporter des solutions constructives. Enfin, nous nous permettons d'insister sur le fait que notre analyse se focalise volontairement sur la phase de conception des PCD dans la mesure où les processus ne sont que très rarement aboutis et, de fait, la phase de conception étant la première, c'est celle qui permet un recueil des données les plus significatives.

\section{La gouvernance du développement local au travers de l'implication des parties prenantes}

Cette recherche fait appel en premier lieu à la notion de développement territorial. Dans ce cadre précis, nous entendons cette notion comme un processus de transformation d'un territoire par une volonté et une action politique. Le territoire est ici compris comme « un système complexe constitué d'éléments continuellement en interaction tels que : le sol, les ressources naturelles, l'habitat, les infrastructures, les entreprises, les individus,

\footnotetext{
${ }^{9}$ Bédard, J ,Gendron, Y., « Qualitative research on accounting: some thoughts on what occurs behind the scene, in Humphrey, C. et Lee, B. (edition) », The real life guide to accounting research: a behind-the-scene view of using qualitative research methods, Elsevier Ltd, 2004

${ }^{10}$ Ahrens, T., Chapman, S., « Doing qualitative Weld research in management accounting: Positioning data to contribute to theory», Accounting, Organization and Society, Elsevier Ltd, Vol. 31; Iss 8, 2006, pp. 819-841
} 
institutions, lois et règlements $»^{11}$. C'est au vu de ces interactions et de leur complexité que nous pouvons affirmer que les processus de développement local au Maroc répondent à une logique dite de « gouvernance ».

Ce concept étant considéré comme un concept polysémique ${ }^{12}$, il convient de restreindre son champ d'application afin de détailler ses principales dimensions applicables à cette recherche. Concernant le secteur public, il est possible de considérer que la gouvernance a deux dimensions principales : elle suppose en premier lieu un nouveau mode d'exercice du pouvoir qui va être plus gestionnaire, plus axé sur la notion de performance que l'on peut comprendre comme «le processus de formation de la perfection ${ }^{13}$. Le concept de gouvernance induit donc une réelle rupture avec le pouvoir central, vertical et hiérarchique. Le terme correspond à "a new process of governing; a changed condition of ordered rules; a new method by which society is governed" ${ }^{\prime 4}$. On passe alors d'une structure hiérarchique du pouvoir à une structure horizontale ce qui a justifié le recours à ce nouveau terme : la gouvernance. En second lieu, elle induit également des liens accrus entre marché, État et société civile. Cela répond à crise de légitimité que l'État connaît depuis la deuxième moitié du XXème siècle : les décideurs politiques et les hauts fonctionnaires étant jugés déconnectés des réalités du quotidien des populations, Le but est de mettre d'avantage les citoyens à contribution dans la décision. Les processus décisionnels deviennent donc plus participatifs, plus proches de la population. A partir de là, on distingue deux principales logiques de gouvernance territoriale: la logique top-down et la logique bottom-up. L'approche la plus répandue et traitée est naturellement celle de la planification stratégique. Cette approche consiste en la formulation de modèles ou de processus de planification qui suivent typiquement une logique top-down. On parle souvent de «market led process », dans lequel un ou deux acteurs principaux font les choix stratégiques et exercent un fort leadership ${ }^{15}$. On a donc potentiellement de nombreuses parties prenantes mais qui ne sont intégrées que dans la phase de réflexion et non dans le processus de décision. Souvent, dans ce cas, les choix stratégiques dépendent de considérations principalement d'ordre économique.

\footnotetext{
11 Geron (G.), « La conférence permanente du développement territorial hier, aujourd'hui demain. Histoire d'un projet », Les Cahiers de l'Urbanisme, n³4, 2001, pp.13-18

12 Baron (C.), «La gouvernance: débats autour d'un concept polysémique », Droit et société, 2003/2, N 54, 2003, pp. 329-349.

${ }^{13}$ Guenoun (M.), Le management de la performance publique locale, Etude de l'utilisation des outils de gestion dans deux organisations intercommunales, Thèse de Doctorat, Université Paul Cézanne Aix-Marseille III, 2009

${ }^{14}$ Rhodes (RAW), " The New Governance: Governing without Government », Political Studies Association, XLIV, 1996, pp. $652-653$.

${ }^{15}$ Flagestad (A.) \& Hope (C. A.), "Strategic Success in Winter Sports Destinations: a Sustainable Value Creation Perspective", Tourism Management, n²2, 2001, pp. 445-461.
} 
Si l'on se réfère aux écrits de Mintzberg $^{16}$, on peut interpréter la stratégie comme le résultat émergeant d'un processus dit bottom-up. Cette perspective constructiviste suppose une forte implication des acteurs d'un territoire lorsqu'il s'agit des choix en termes de développement. Le processus de définition de la stratégie territoriale émerge alors d'une mise en réseau des acteurs ${ }^{17}$. Le processus de décision est donc très collaboratif, et fonctionne grâce à l'emploi d'outils tels que les tables rondes, les assemblées, et tous les autres instruments encourageant la participation ainsi que sur des mécanismes formels de planification $^{18}$. Cette logique est privilégiée lorsqu'on prend en compte la dimension sociale dans le développement territorial ${ }^{19}$. Selon Jamal et $\mathrm{Getz}^{20}$, le succès d'une telle initiative dépend strictement du développement d'une vision partagée et d'objectifs communs dans la planification stratégique et donc de la création d'une « organisation coopérative stable ».

Ces deux approches ne sont pas pour autant nécessairement dichotomiques. Stokes ${ }^{21}$, en se basant sur ces dernières, en a défini une troisième : « the synergistic model ». Ce modèle combine les buts, priorités et processus des deux modèles précédents. En effet, selon lui, la gouvernance territoriale cherche à atteindre un équilibre entre les besoins des acteurs publics, ceux des entreprises et ceux de la communauté locale en utilisant une stratégie combinant des processus communautaires de consultation de type bottom-up avec des processus de décision basés sur la logique top-down. De fait, elle attribue dans les décisions un poids similaire aux dimensions économiques et aux dimensions socio-culturelles et environnementales. Concernant le développement local au Maroc, nous nous réfèrerons principalement à la théorie dominante concernant l'organisation des processus de développement dans le monde et plus spécifiquement au Maroc : celle de la « bonne gouvernance $»^{22}$ comme l'affirment la section marocaine du PNUD et le Ministère du développement social de la famille et de la

\footnotetext{
${ }^{16}$ Mintzberg (H.), “The rise and fall of strategic planning”, Harvard Business Review, n72, 1994, pp. 107 114 ,

17 Halme, M., "Learning for sustainable development in tourism networks", Business Strategy and the Environment, $\mathrm{N}^{\circ} 10: 2,2001$, pp. 101-113.

18 Getz (D.) et Jamal (T.), « Does strategic planning pay? Lessons for destinations from corporate planning experience », Progress in Tourism and Hospitality Research, ${ }^{\circ} 2$, 1995, pp. 59- 78

${ }_{19}$ Bonetti (E.), "Combining the top-down and bottom-up approach: the morphing characteristics of the territorial governance”, European Academy of Management conference, 2011.

${ }^{20} \mathrm{Jamal}$ (T.), Tazim (B.) et Getz (D.) "Community round tables for tourism related conflicts: the dialectic of consensus and process structures", in Bill Bramwell and Bernard Lane (eds.), Tourism collaboration and partnerships, Clevedon: Channel View Publications, 2000, pp 159-182.

${ }^{21}$ Stokes (R.), "Tourism strategy making: insights to the events tourism domain”, Tourism Management, $\mathrm{n}^{\circ} 29$, 2008, pp. 252-262.

22 « Governance and development», World Bank's policy statement, 1992.
} 
solidarité dans leur rapport de février $2005^{23}$. Elle prône, dans la conception des plans de développement locaux, une approche participative de type bottom-up ${ }^{24}$ bien que les outils managériaux du système émanent d'une conception de l'État dans une logique top-down. Le processus de conception est alors participatif à l'échelon local mais implique également l'État à différents niveaux de déconcentration du pouvoir. De surcroit, même si les différentes étapes de la conception du plan répondent à des impératifs de gouvernance participative, il est important de préciser que le plan est dans tous les cas avalisé par les échelons déconcentrés supérieurs avant toute possibilité de mise en œuvre. Nous sommes donc ici dans une conception qui se rapproche clairement du « synergistic model » de Stokes que nous retenons donc comme prisme de lecture. Dans tous les cas, le concept de gouvernance est donc, dans cette dimension comme à son origine, intrinsèquement lié à la question des parties prenantes du processus. En effet, "The developmental processes of a territory are complex. In fact, the large number of stakeholders involved makes the development dynamics of an aera far from linear" 25 .

L'approche sur la théorie des parties prenantes ou Stakeholder Theory prend véritablement ses racines dans le travail de Berle et Means $(1932)^{26}$. Les auteurs démontrent l'impact social sur les décisions de l'entreprise de tous ceux dont le bien-être peut être affecté. Plut tard ces derniers ont été déterminés comme « groupe qui dépend de l'entreprise pour réaliser ses buts propres et dont cette dernière dépend pour assurer son existence » (Rhenman et Stymne, $1965^{27}$ ) ou comme « tout groupe dont le comportement collectif peut affecter directement l'avenir de l'organisation, mais qui n'est pas sous le contrôle direct de celle-ci » (Sturdivant, 1979 , p. $54^{28}$ ). Les parties prenantes, en effet, représentent « groupes d'intérêt, parties, acteurs, prétendants et institutions (tant internes qu'externes) qui exercent une influence sur l'entreprise. Parties qui affectent ou sont affectées par les actions, comportements et politiques de l'entreprise » (Mitroff, 1983, p. $4^{29}$ ). Manal El Abboubi et

\footnotetext{
23 PNUD Maroc, «Ensemble pour un développement humain : Pour une politique de développement social intégré », Rapport de mission d'appui du PNUD au Ministère du Développement Social de la Famille et de la Solidarité du Royaume du Maroc, Février 2005.

${ }^{24}$ Mintzberg (H.), Grandeur et décadence de la planification stratégique, Dunod, Paris, 1994

25 Bonetti (E.), "Combining the top-down and bottom-up approach: the morphing characteristics of the territorial governance", European Academy of Management conference, 2011, p. 2.

${ }_{26}$ Berle (A. A.) et Means (G.), The Modern Corporation and the Private Property, New York, Mc Millan,1932

${ }^{27}$ Rhenman (E.) \& Stymne (B.), Corporate Management in a Changing World, Aldus/Bonniers, Stockholm. 1965

${ }^{28}$ Sturdivant (F. D.), "Executives and Activists: Test of Stakeholder Theory", California Management Review, vol. 22, $\mathrm{n}^{\circ} 1,1979$, pp. 53-59.

${ }^{29}$ Mitroff (I.), Stakeholders of the Organizational Mind, Jossey-Bass, San Francisco,1983
} 
Annie Cornet $(2010)^{30}$ résument les différentes approches par les parties prenantes (tableau 1). Selon les auteurs, il existe sept catégories de parties prenantes. La première, appelée relationnelle, est surtout représentée par les travaux fameux de Freeman (Freeman, 1984; Freeman \& Reed, $1983^{31}$ ) qui décrivent les relations entre les organisations et les acteurs du marché et étudient l'influence de ces derniers. Clarkson (Clarkson, 1995 ${ }^{32}$ ) représentait l'approche juridique avec les notions de propriétés, de droits et d'intérêts. Mitchell et al. enrichissent les théories existantes avec une approche : l'attribut. Selon les auteurs, les parties prenantes « possèdent au moins un de ces trois attributs : le pouvoir, la légitimité et l'urgence » (Mitchell, Agle et Wood, 1997).

\begin{tabular}{|c|c|c|}
\hline Approche & Idée principale & Auteurs / Sources \\
\hline Relationnelle & $\begin{array}{l}\text { Les parties prenantes sont celles qui ont une relation } \\
\text { avec l'organisation. }\end{array}$ & $\begin{array}{l}\text { (Freeman, } 1984 \text {; Freeman \& Reed, } \\
1983 \text {; Rhenman \& Stymne, 1965) }\end{array}$ \\
\hline Contractuelle & $\begin{array}{l}\text { Les parties prenantes sont celles qui ont une relation } \\
\text { contractuelle avec l'organisation. }\end{array}$ & (Cornell \& Shapiro, 1987) \\
\hline Légitimité & $\begin{array}{l}\text { Les parties prenantes sont celles qui sont perçues } \\
\text { comme légitimes par l'organisation. } \\
\text { Le lien peut être sous la forme d'une relation } \\
\text { contractuelle ou non. }\end{array}$ & (Donaldson \& Preston, 1995) \\
\hline Droits & $\begin{array}{l}\text { Les parties prenantes sont celles qui ont des droits ou } \\
\text { intérêts en regard de l'organisation. }\end{array}$ & $\begin{array}{l}\text { (Clarkson, 1995; Freeman \& Evan, } \\
\text { 1990; Hill \& Jones, 1992) }\end{array}$ \\
\hline Contributions & $\begin{array}{l}\text { Les parties prenantes sont celles qui apportent une } \\
\text { contribution à l'organisation. } \\
\text { La forme de la contribution peut varier. }\end{array}$ & $\begin{array}{l}\text { (Clarkson, 1995; Wicks, Gilbert \& } \\
\text { Freeman, 1994) }\end{array}$ \\
\hline Attributs & $\begin{array}{l}\text { Les parties prenantes sont celles qui ont certains } \\
\text { attributs tels que le pouvoir, la légitimité ou l'urgence. }\end{array}$ & (Mitchell, et. al., 1997) \\
\hline Engagement & $\begin{array}{l}\text { Les parties prenantes sont celles qui ont un engagement } \\
\text { Sociétal ou organisationnel. }\end{array}$ & (Girard \& Sobczak, 2010) \\
\hline
\end{tabular}

Tableau 1: Catégorisation des parties prenantes proposée par Manal El Abboubi et Annie Cornet (2010, p.277)

Une des nouvelles approches développée en 2010 par Girard et Sobczak (2010) et appelée «l'engagement» insiste sur le fait que les parties prenantes doivent s'engager sociétalement ou organisationnellement. Les auteurs proposent une classification des parties prenantes selon deux axes : l'implication organisationnelle et l'implication sociale, qui donnent quatre catégories : les parties prenantes alliées, engagées, passives et militantes.

\footnotetext{
${ }^{30}$ El Abboubi (M.) \& Cornet (A.), « L'implication des parties prenantes comme un processus de construction sociale. Analyse à partir de la théorie de l'acteur-réseau », Revue Management et Avenir, n³3, 2010, pp. 275296.

${ }^{31}$ Freeman R. E. et D. L. Reed, "Stockholders and Stakeholders: A New Perspective on Corporate Governance", California Management Review, vol. 25, n 3, spring 1983, p. 88-106.

${ }_{32}$ Clarkson (M. B.), A Stakeholder Framework for Analyzing and Evaluating Corporate Social Performance, Academy of Management Review, N²0 : 11, 1995, pp. 92-117.
} 
En effet, il existe plusieurs classifications des parties prenantes. La classification la plus connue et la plus utilisée a été proposée par Carol $(1989)^{33}$. L'auteur distingue les parties prenantes primaires et les parties prenantes secondaires. Par les parties prenantes primaires encore qualifiées de parties prenantes contractuelles l'auteur distingue les acteurs qui ont des relations contractuelles avec l'organisation. Les parties prenantes secondaires qualifiées de diffuses réunissent les acteurs ayant un impact sur l'entreprise qui n'ont pas de lien contractuel avec l'organisation.

Pour cette recherche nous nous sommes intéressés à la classification proposée par Pelle Culpin $(1998)^{34}$. Nous avons retenu cette classification car elle permet une catégorisation précise et pertinente des différents types d'acteurs présents sur le territoire. En effet l'auteur a distingué les parties prenantes institutionnelles, les parties prenantes économiques et enfin les parties prenantes éthiques. Les parties prenantes institutionnelles regroupent les acteurs liés aux lois, réglementations qui appartiennent aux organisations professionnelles et institutionnelles. Les parties prenantes économiques incluent les acteurs opérant sur les marchés. Enfin, les parties prenantes éthiques réunissent les acteurs ayant un impact sur l'organisation par pression éthique et politique. Bien évidemment, ces derniers ne sont pas faciles à déterminer.

L'objectif de notre travail est d'identifier les parties prenantes impliquées. Pour réaliser cela, il nous semble opportun d'appliquer la théorie de l'acteur-réseau (Callon, $\left.1986^{35}\right)$. En effet, cette théorie a d'ores et déjà été utilisée comme support d'analyse du processus de mobilisation des parties prenantes par exemple dans les travaux de Manal El Abboubi et Annie Cornet (2010). Selon ces auteurs : «cette théorie est très éclairante pour comprendre le comportement des acteurs et pour isoler les éléments qui vont susciter ou, au contraire, bloquer l'adhésion au projet de certification de l'organisation » (p. 280). Comme ces auteurs, nous n'allons utiliser qu'une partie de la théorie de l'acteur-réseau à savoir les phases de contextualisation (qui nous permettra d'identifier les parties prenantes mobilisées, d'analyser leurs intérêts et leurs enjeux autour de la conception d'un plan communal de développement), de problématisation (qui va nous aider à comprendre comment le porteur de

\footnotetext{
33 Caroll A. B., Business and Society: Ethics and Stakeholder Management, O.H.: South Western, Cincinatti, 1989

${ }^{34}$ Pelle Culpin (C. I.), Du paradoxe de la diffusion d'informations environnementales par les entreprises européennes, Thèse de Doctorat, Université de Paris IX Dauphine, 1998

${ }^{35}$ Callon (M.), «Éléments pour une sociologie de la traduction, la domestication des coquilles saint-jacques et des marins-pêcheurs dans la baie de Saint-Brieuc », L'Année Sociologique, Vol. 36, 1986, p. 169-208.
} 
projet a formulé le projet pour obtenir la coopération des parties prenantes) et de mobilisation (dont l'objectif est de définir les rôles que chacun joue dans l'action et le projet étudié). (Akrich, Callon et Latour, 2006 ${ }^{36}$ ).

${ }^{36}$ Akrich (M.), Callon (M.), Latour (B.), Sociologie de la traduction. Textes fondateurs, Paris, Presses de l'École des Mines de Paris, 2006 
Schéma récapitulatif du cadre conceptuel

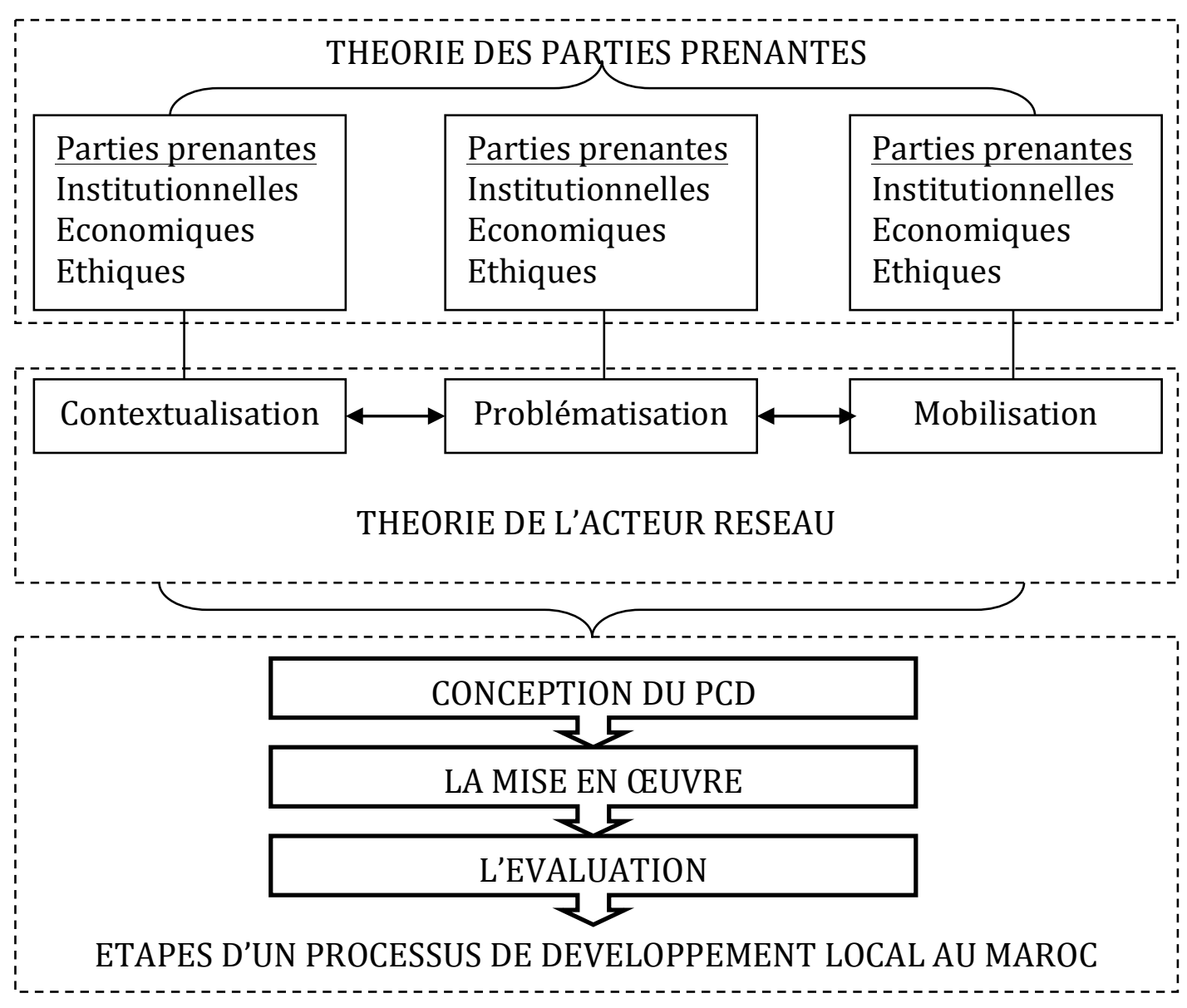

Schéma élaboré à partir des travaux d'Akrich, Callon et Latour (2006) et Manal El Abboubi et Annie Cornet $(2010)^{37}$

37 El Abboubi, M, \& Cornet, A., «L'implication des parties prenantes comme un processus de construction sociale : analyse à partir de la théorie de l'acteur-réseau », Revue Management et Avenir, N 33, 2010, pp. 275 à 297. 


\section{Méthodologie et terrain de recherche}

Notre problématique de recherche, étant relativement peu traitée dans la littérature, une étude qualitative exploratoire est préférable puisqu'elle permettra de saisir la richesse des données (Bédard et Gendron, 2004 ${ }^{38}$; Ahrens et Chapman, 2006 ${ }^{39}$ ). Ce type d'étude a pour objectif une meilleure compréhension des phénomènes se réalisant dans leur contexte naturel (Berry et al. 2004 ${ }^{40}$ ) à savoir dans un environnement qui n'est ni contrôlé, ni perturbé ou orienté par les postulats du chercheur. On cherche alors à définir l'objet de recherche en le faisant émerger du terrain par une compréhension et une analyse les plus objectives possibles des expériences et réalités quotidiennes des acteurs et des organisations étudiées. La compréhension concerne le pourquoi et le comment des phénomènes et des événements dans des situations concrètes (Wacheux, $1996^{41}$ ).

La méthode de recueil des données retenue est l'entretien d'expert. Au nombre de huit, ces entretiens seront analysés textuellement par une technique de double codage liée aux deux pans de notre revue de littérature. Comme terrain de recherche, pour les raisons précédemment énoncées, nous avons choisi le Maroc.

Depuis l'adoption de la Charte Communale en 2002 et, dans son prolongement, depuis l'Initiative Nationale pour le Développement Humain lancée par SM le Roi Mohammed VI en 2005, chaque commune marocaine est légalement contrainte de se doter d'un Plan Communal de Développement afin d'envisager son avenir en termes de développement local. De fait, toutes les communes du pays se sont lancées peu à peu dans la conception de ces fameux PCD de façon à se doter d'un document permettant de faire émerger les grands axes de développement de chaque territoire. Il est nécessaire ici de préciser que les PCD sont, du fait de la taille des territoires concernés, des plans globaux transsectoriels qui permettent d'axer la réflexion et l'action publique sur les éléments ayant le plus de potentiel. A donc émergé un processus complexe qui part de la prise de décision du Conseil Communal de se lancer dans la

\footnotetext{
${ }^{38}$ Bédard, J., et Gendron, Y., « Qualitative research on accounting: Some thoughts on what occurs behind the scene ", The Real Life Guide to Accounting Research, eds. C. Humphrey et B. Lee, Londres, Angleterre: Elsevier, 2004, pp. 191- 206

${ }^{39}$ Ahrens T. and Chapman C. S., «Doing qualitative field studies: positioning data to contribute to theory », Accounting, Organizations and Society, $\mathrm{N}^{\circ} 31(8), 2006$, pp. 819-841

40 Berry, A. J et Otley, D. T., «Case-based research in accounting », pp. 231-255, 2004, in Humphrey, C. et Lee, B. (edition), The real life guide to accounting research: a behind-the-scene view of using qualitative research methods, Elsevier Ltd.

${ }^{41}$ Wacheux, F., Méthodes Qualitatives et Recherche en Gestion, Paris: Economica, 1996
} 
conception du PCD et qui aboutit normalement à l'évaluation de la mise en œuvre des différentes actions prévues dans le plan.

On peut globalement identifier trois grandes étapes :

- La première est celle de la conception du PCD.

- La seconde est celle de la mise en œuvre

- La troisième est celle de l'évaluation

Cette recherche ayant pour objectif d'analyser la conception des plans communaux de développement au Maroc afin d'observer l'implication des différentes parties prenantes, nous avons volontairement choisi de cantonner notre analyse à l'étape de conception même si, pour le bien de la discussion et l'ouverture sur des voies de recherche ultérieures, nous nous permettrons d'aborder succinctement la question du passage de la conception à la mise en œuvre ainsi que celle des évaluations.

S'agissant de la phase de conception, cette étude cherche à la fois, d'un point de vue descriptif, à identifier les acteurs impliqués dans la conception des PCD mais aussi à étudier leurs relations notamment en termes de distribution du pouvoir. D'autre part, le caractère exploratoire de cette étude nous a également poussé à nous interroger aux éléments qui sont plutôt favorables à la conception des PCD mais aussi à ceux pouvant aboutir, dans certaines configurations, à l'échec des processus.

Pour répondre à toutes ces questions, nous avons opté pour une méthodologie qualitative qui, selon Evrard, Roux et al. (2006) ${ }^{42}$, se justifie lorsque les champs d'études sont nouveaux et/ou complexes : l'entretien d'expert. Nous avons considéré comme experts, un certain nombre de chargés de missions ou directeurs en bureaux d'études techniques chargés de la conception de PCD; des élus locaux ayant eu à en piloter, des membres des ministères impliqués dans la conception de plusieurs plans (notamment des hauts fonctionnaires du Haut Commissariat au Plan) et des universitaires réalisant des missions d'expertises en développement au Maroc.

La spécificité de cet échantillon n'est pas, contrairement à l'usage en Sciences de Gestion, une dimension territoriale dans la mesure où certains oeuvrent dans le nord du pays et d'autres dans le sud. Cependant, compte tenu de la standardisation à l'échelle nationale du processus de conception des PCD, on peut considérer qu'ils sont tous experts, dans des domaines différents, du même sujet. Il s'agit donc d'une étude de cas unique : la conception des PCD au Maroc, interprétée sous le prisme de la répartition des parties prenantes.

\footnotetext{
${ }^{42}$ Evrard, Pras, Roux et al. Market, Etudes et Recherches en Marketing, Dunod, 2009
} 
Le tableau ci-dessous détaille le statut professionnel des répondants, la durée de la période où ils ont participé à la conception de plans de développement ainsi que leur nationalité. L'anonymat est, conformément à leurs demandes respectives du fait de leur haut niveau de responsabilités, naturellement conservé. Aussi, les répondants sont numérotés aléatoirement et nous restons volontairement vagues quant à la description de leurs fonctions respectives.

\begin{tabular}{|c|c|c|c|c|c|}
\hline Répondants & Fonctions & $\begin{array}{l}\text { Période } \\
\text { d'exercice }\end{array}$ & Nationalité & $\begin{array}{l}\text { Age du } \\
\text { répondant }\end{array}$ & $\begin{array}{l}\text { Durée de } \\
\text { l'entretien }\end{array}$ \\
\hline$N^{\circ} 1$ & $\begin{array}{l}\text { Directeur de la branche } \\
\text { "développement » d'un } \\
\text { bureau d'études } \\
\text { spécialisé secteur public }\end{array}$ & $2001-2013$ & Marocaine & 51 ans & $1 \mathrm{~h} 13$ \\
\hline $\mathrm{N}^{\circ} 2$ & $\begin{array}{l}\text { - Élue locale } \\
\text { - Vice-présidente de } \\
\text { Conseil Communal } \\
\text { - Chercheur en Sciences } \\
\text { de Gestion }\end{array}$ & $\begin{array}{l}2004-2013 \\
2009-2013 \\
2009-2013\end{array}$ & Marocaine & 58 ans & $1 \mathrm{~h} 24$ \\
\hline$N^{\circ} 3$ & $\begin{array}{l}\text { - Directeur régional du } \\
\text { Haut Commissariat au } \\
\text { Plan } \\
\text { - Expert en bureaux } \\
\text { d'études }\end{array}$ & $\begin{array}{l}1982-2005 \\
2005-2013\end{array}$ & Marocaine & 59 ans & $48 \mathrm{~min}$ \\
\hline $\mathrm{N}^{\circ} 4$ & $\begin{array}{l}\text { - Chargée de mission } \\
\text { « développement » en } \\
\text { bureau d'études }\end{array}$ & $2005-2013$ & Française & 29 ans & $1 \mathrm{~h} 08$ \\
\hline$N^{\circ} 5$ & $\begin{array}{l}\text { - Président d'une } \\
\text { association chargée de } \\
\text { concevoir des PCD au } \\
\text { Maroc }\end{array}$ & $2002-2013$ & Française & 38 ans & $41 \mathrm{~min}$ \\
\hline $\mathrm{N}^{\circ} 6$ & $\begin{array}{l}\text { - Wali d'une des régions } \\
\text { du Maroc }\end{array}$ & $1999-2005$ & Marocaine & 54 ans & $53 \mathrm{~min}$ \\
\hline$N^{\circ} 7$ & $\begin{array}{l}\text { - Professeur en droit } \\
\text { public } \\
\text { - Expert en } \\
\text { développement } \\
\text { territorial }\end{array}$ & $1985-2013$ & Marocaine & 63 ans & $1 \mathrm{~h} 03$ \\
\hline$N^{\circ} 8$ & $\begin{array}{l}\text { - Membre à haut niveau } \\
\text { de responsabilités de la } \\
\text { Direction Générale des } \\
\text { Collectivités Locales }\end{array}$ & $1993-2013$ & Marocaine & 49 ans & $1 \mathrm{~h} 18$ \\
\hline
\end{tabular}

Tableau 2 : Échantillon et expertise des répondants

\section{Résultats}

Pour la présentation de nos résultats, nous avons opté pour une approche en deux temps. Premièrement, nous proposons une approche qui découle de l'application de notre modèle. Nous allons donc présenter ici nos résultats selon trois axes à savoir la contextualisation qui prendra la forme d'une identification des acteurs impliqués dans la conception des PCD, la 
problématisation qui présentera les sources des PCD en termes d'initiatives et enfin, la mobilisation qui présentera le niveau d'implication de chaque acteur présenté dans la contextualisation. Dans un second temps, nous présenterons d'autres éléments émergeant de notre étude exploratoire et que nous pouvons considérer comme émanant de la mise en relation des différentes parties prenantes.

1. Application du modèle

Contextualisation et identification des acteurs :

Nous rappelons en préambule que la contextualisation a pour objet de permettre d'identifier les différents acteurs et de les classer selon trois catégories développées par Pelle Culpin (1998) à savoir les acteurs institutionnels, les acteurs économiques et les acteurs éthiques.

Concernant les acteurs institutionnels, une saturation théorique - soit «le moment à partir duquel l'apprentissage incrémentiel est minime, les chercheurs observant des phénomènes déjà constatés $»^{43}$ - de cent pour cent nous permet d'affirmer qu'ils sont les suivants : le Conseil Communal, le Conseil Provincial, les agences et offices déconcentrés comme l'Office National de l'Électricité (ONE), l'Office National de 1'Eau Potable (ONEP) etc.; les représentants des ministères concernés par le développement spécifique de ce territoire comme par exemple le Haut Commissariat aux Eaux et Forêts et à la Lutte Contre la Désertification (HCEFLCD), le Haut Commissariat au Plan, le Ministère des Habous et des Affaires Islamiques, le Ministère de l'Agriculture etc.

On constate également qu'une place particulière est accordée aux ONG que les experts classent comme organismes privés aux cotés des bureaux d'études : "Au niveau privé, si on peut dire, parfois tu as des ONG ou le PNUD qui interviennent pour donner leur avis. Enfin, il y a aussi les bureaux d'études. 》 (Répondant $\mathrm{n}^{\circ} 4$ ).

Enfin, naturellement, la dimension participative obligatoire dans la conception des PCD implique de situer dans cette catégorie, la société civile.

«Q : La société civile est elle vraiment un acteur clef?

$\mathrm{R}$ : J'aimerais qu'il soit (un acteur) clef mais dans la réalité, ça n'est pas le cas. On ne prend pas assez en compte leur avis. Ça c'est quelque chose qui n'est pas ancré je pense. Faire participer la société civile, même si on essaie de le faire car c'est obligatoire, ce n'est pas encore vraiment le cas. (Répondant $\mathrm{n}^{\circ} 5$ )

\footnotetext{
${ }^{43}$ Hlady-Rispal (M.), La méthode des cas : application à la recherche en sciences de gestion, De Boeck, 2002.
} 
$\mathrm{R}$ : Tel que le guide de la DGCL a été conçu, la société civile est impliquée à de nombreuses étapes des processus. Dans les faits, elle est très difficile à mobiliser et dans une dynamique de performance, nous avançons sans elle». (Répondant $\mathrm{n}^{\circ} 8$ )

S'agissant des acteurs économiques, on pourrait croire qu'ils sont inexistants dans la mesure où les financements sont publics. Cependant, la conception des PCD étant par essence complexe et les acteurs n'y étant pas formés (notamment le conseil communal), les collectivités ont souvent recours à des bureaux d'études ou à des associations travaillant avec des experts par le biais d'appels d'offres. De fait, dans ces situations, nous pouvons les considérer comme des acteurs économiques.

«Lorsqu'on construit un PCD, il est impératif de faire appel à un bureau d'études ou à une association qui travaille avec des experts afin de concevoir un plan sérieux. Sinon, on se rend compte que les PCD sont défaillants et qu'ils ne sont jamais mis en æuvre. » (Répondant $\mathrm{n}^{\circ}$ 5)

«Aujourd'hui, la réalité du terrain est que le passage par un appel d'offre pour externaliser la gestion de la conception des PCD est une pratique courante voire même systématique.» (Répondant $\mathrm{n}^{\circ} 6$ )

Pour ce qui est des acteurs éthiques, nous avons regroupé ici les acteurs mis à contribution pour avis consultatif avant la mise en œuvre du plan. N'étant dotés d'aucun pouvoir décisionnel en la matière, ils sont consultés souvent en raison de leur statut honorifique ou de leur expérience en matière de développement local. Ainsi, certaines Organisations Non Gouvernementales, des éminences grises de la société civile ou encore les représentants locaux du Haut Commissariat au Plan sont souvent interrogés quant à la pertinence du plan final avant qu'il soit présenté au Conseil Communal puis au Gouverneur de la Province pour être avalisé définitivement.

Problématisation et processus de mobilisation :

Il s'agit ici d'étudier le processus de mobilisation des acteurs autour d'un projet commun à savoir la conception d'un Plan Communal de Développement. Cependant, dans ce contexte, les PCD émanant d'une volonté nationale, ils s'imposent de fait à tous les acteurs qui vont y être impliqués. Malgré tout, la décision en termes d'organisation du processus revient au conseil communal. Il faut noter que les PCD s'inscrivent souvent dans le cadre d'un plan provincial qui est ventilé par la suite au niveau communal. La dynamique est donc impulsée par la province mais l'organisation de la conception de chaque PCD relève du Conseil Communal. Aussi, comme nous en ont informé l'ensemble des personnes interrogées 
lors de notre étude de terrain, même si le plan est obligatoire au sens où même si la commune ne le met pas en œuvre, elle doit être dotée d'un document contenant le PCD, il y a une dimension qualitative à prendre en compte. En effet, nombreux sont les plans conçus à la vavite et qui ne seront jamais mis en œuvre. A contrario, lorsque le plan est construit «dans les règles de l'art» (Répondant $\mathrm{n}^{\circ} 3$ ), c'est «l'implication, le pilotage et le leadership du Président du Conseil Communal qui l'ont permis » (Répondant n³). C'est pourquoi, nous considérons ici que l'obligation légale ne fait pas tout et que même si elle existe, la réalisation d'un document de qualité et, dans son prolongement, le passage de la conception à la mise en œuvre ne saurait se passer d'une forte implication des élus de la commune afin de fédérer à la fois les acteurs institutionnels mais aussi la société civile autour d'un projet de grande envergure.

Mobilisation et niveau d'implication des acteurs

Nous avons ici structuré notre analyse en termes de niveaux d'implication des acteurs. Nous les avons donc répartis dans le tableau suivant en fonction de leurs rôles respectifs ainsi que de leur catégorie (institutionnels, économiques ou éthiques)

\begin{tabular}{|c|c|c|c|}
\hline & Acteurs institutionnels & Acteurs économiques & Acteurs éthiques \\
\hline $\begin{array}{l}\text { Acteurs chargés du } \\
\text { pilotage de la } \\
\text { conception }\end{array}$ & - Conseil communal & & \\
\hline $\begin{array}{l}\text { Acteurs détenant le } \\
\text { pouvoir décisionnel }\end{array}$ & $\begin{array}{l}\text { - Conseil communal } \\
\text { - Conseil provincial }\end{array}$ & & \\
\hline $\begin{array}{l}\text { Acteurs chargés de } \\
\text { déterminer les axes } \\
\text { stratégiques et objectifs } \\
\text { par secteur }\end{array}$ & $\begin{array}{l}\text { - Société civile } \\
\text { - Représentants des } \\
\text { ministères concernés } \\
\text { - Représentants locaux } \\
\text { des agences et offices } \\
\text { déconcentrés }\end{array}$ & $\begin{array}{l}\text { - Bureau d'études ou } \\
\text { associations }\end{array}$ & \\
\hline $\begin{array}{l}\text { Acteurs ayant un avis } \\
\text { consultatif }\end{array}$ & & & $\begin{array}{l}\text { - ONG } \\
\text { - Représentants du } \\
\text { Haut Commissariat au } \\
\text { Plan } \\
\text { - Membres éminents } \\
\text { de la société civile } \\
\end{array}$ \\
\hline $\begin{array}{l}\text { Acteurs chargés de la } \\
\text { formation et apportant } \\
\text { des ressources } \\
\text { humaines et matérielles }\end{array}$ & $\begin{array}{l}\text { - Commune (ressources } \\
\text { financières) } \\
\text { - Province (ressources } \\
\text { financières) } \\
\text { - Ministères, agences et } \\
\text { offices (ressources } \\
\text { documentaires) }\end{array}$ & $\begin{array}{l}\text { - Bureau d'études ou } \\
\text { associations } \\
\text { (ressources humaines } \\
\text { et matérielles) }\end{array}$ & \\
\hline
\end{tabular}

Tableau 3 : Rôle des acteurs en fonction de leur catégorie en tant que partie-prenante 
2. Éléments émanant de la mise en relation des acteurs

Comme sus-énoncé, de notre terrain ont également émergé des éléments significatifs. Le premier d'entre eux qui est revenu de façon récurrente dans l'ensemble de nos entretiens est le problème de la formation des élus que ce soit au pilotage des PCD ou à la logique même de développement local. En effet, selon les experts, « la logique de développement local dans un État à la structure si concentrée a du mal à s'ancrer. » (Répondant $\mathrm{n}^{\circ} 3$ ). «Ils ne voient même pas l'intérêt de concevoir un PCD» (Répondant $\mathrm{n}^{\circ} 7$ ). S'agissant du pilotage, il est important de considérer que les élus ne sont pas formés en amont. "Il existe des formations mais elles ne sont pas obligatoires alors soit ils n'y vont pas, soit c'est jamais le même qui vient et donc nos enseignements n'ont plus de cohérence » (Répondant $\mathrm{n}^{\circ} 4$ ). «On est formé au pilotage en même temps qu'on pilote. Imaginez un avion plein de gens dont le pilote n'a même jamais appris la théorie et encore moins piloté, comment voulez vous que les gens arrivent en vie à destination? $\gg$ (Répondant $\mathrm{n}^{\circ} 2$ ).

Le second résultat qui nous a paru intéressant à développer est celui du manque d'implication de certains acteurs et de ses causes. En effet, selon nos experts, «souvent, le problème c'est que la société civile et le Conseil Communal n'en ont rien à faire. Ils ne voient pas l'intérêt». (Répondant $\mathrm{n}^{\circ} 1$ ). "On aimerait que la société civile soit plus mise à contribution mais ils s'en foutent complètement car ils ne voient pas en quoi ça va changer leur situation » (Répondant $\mathrm{n}^{\circ}$ ). En recherche des raisons de ce manque d'implication, nous les avons interrogés et la réponse est unanime. S'agissant de la société civile, il semblerait qu'une désillusion totale quant à la capacité des pouvoirs publics à améliorer la situation soit en cause. " A force de se voir promettre la lune et de ne voir la situation qu'empirer, ils n'y croient plus» (Répondant $\left.\mathrm{n}^{\circ} 4\right)$. «Selon moi, on a une vraie crise de légitimité de l'État comme des gouvernements locaux qui induit que les populations n'ont plus confiance en les institutions publiques pour améliorer leur situation » (Répondant $\mathrm{n}^{\circ} 2$ ). Concernant les élus, d'autres éléments sont à prendre en compte. Il semblerait que la question des intérêts partisans soit en cause. En effet, selon nos répondants, les élus se trouvent plus souvent dans des considérations électoralistes, ethniques ou financières que dans une logique d'intérêt général. «Vous voyez, il y a des communes où le président de commune s'en fiche carrément et qui n'a que des objectifs électoraux. Donc ce qui lui importe c'est de faire tout ce qui se verra en vue de gagner les élections. Juste pour la façade et que les gens se disent qu'il bosse. » (Répondant $\mathrm{n}^{\circ} 1$ ). «Le Président d'une commune rurale comme la nôtre est là plus pour le 
prestige que pour mettre en place des mécanismes de bonne gestion, de bonne gouvernance locale comme veut bien le véhiculer le mot d'ordre du ministère de l'intérieur » (Répondant $\left.\mathrm{n}^{\circ} 2\right)$.

\section{Discussion conclusive}

Dans ce travail de recherche, nous avons montré l'implication des parties prenantes dans la conception des plans communaux de développement au Maroc. L'originalité de notre projet de recherche réside dans la combinaison des modèles traditionnels d'analyse du positionnement des parties prenantes (Mitchell, et al., 1997; Girard et Sobczak, 2010) avec une analyse du processus de mobilisation à l'aide de la théorie de l'acteur-réseau (Akrich et al., 2006). Notre analyse, à destination des pouvoirs publics, a pour objet d'aider à l'amélioration de la gestion de cette phase des processus de développement territorial. Les premiers résultats de notre recherche ont révélé le problème de la formation des élus. L'ensemble des experts interrogés insiste sur le fait que le manque de formation est devenu la cause principale de défaillance dans la conception des PCD. En effet le problème de la formation des élus est reconnu comme un objet de recherche depuis des années. Les auteurs remarquent que « la formation des élus locaux se présente comme un système éclaté, reposant sur une variété d'initiatives nationales et locales » (Maisl et Pouyet, 1981 ${ }^{44}$ ). Une intégration plus forte de la formation des élus aidera « à assurer une amélioration de la capacité collective à anticiper les problèmes, à les identifier et à réagir rapidement, ainsi qu'à assurer un décloisonnement de l'organisation, une meilleure cohérence et adaptation des actions vis-àvis du projet d'ensemble poursuivi » (Demeestère, 1996, p.59 $9^{45}$ ). Une formation plus adaptée des élus permettra donc d'améliorer chaque étape du processus de conception des plans communaux de développement au Maroc et simplifiera l'intervention avec les parties prenantes. Au regard de nos résultats, il nous semble essentiel de souligner que la formation, au delà de la dimension technique des PCD, doit en amont porter sur l'intérêt de rentrer dans une logique de développement local. En effet, le résultat le plus significatif et intéressant de cette recherche est, selon nous, le manque de considération de ces processus de la part des élus qui, pour citer nos experts «n'en voient même pas l'intérêt ». Cette réalité semble être liée à la dimension top-down de l'initiative des processus qui sont imposés aux élus locaux par l'État central et qui les amènent à les déconsidérer. Il faudrait donc qu'ils s'approprient la

\footnotetext{
${ }^{44}$ Maisl (H.), Pouyet (B.), Annuaire des collectivités locales, Persée, 1981

${ }^{45}$ Demeestère (R.), « Quelle démarche de pilotage pour une collectivité territoriale ? », Revue Française de Finances Publiques, N53, 1996, pp. 55-60.
} 
démarche elle-même avant de pouvoir piloter efficacement les processus. Concernant nos voies de recherches ultérieures, nous considérons qu'il serait également intéressant d'analyser le processus intégral allant de la conception à l'évaluation en passant par la mise en œuvre afin de pouvoir comparer l'implication des parties prenantes à chaque étape et notamment la place investie, ou non, par le Conseil Communal et son Président. 